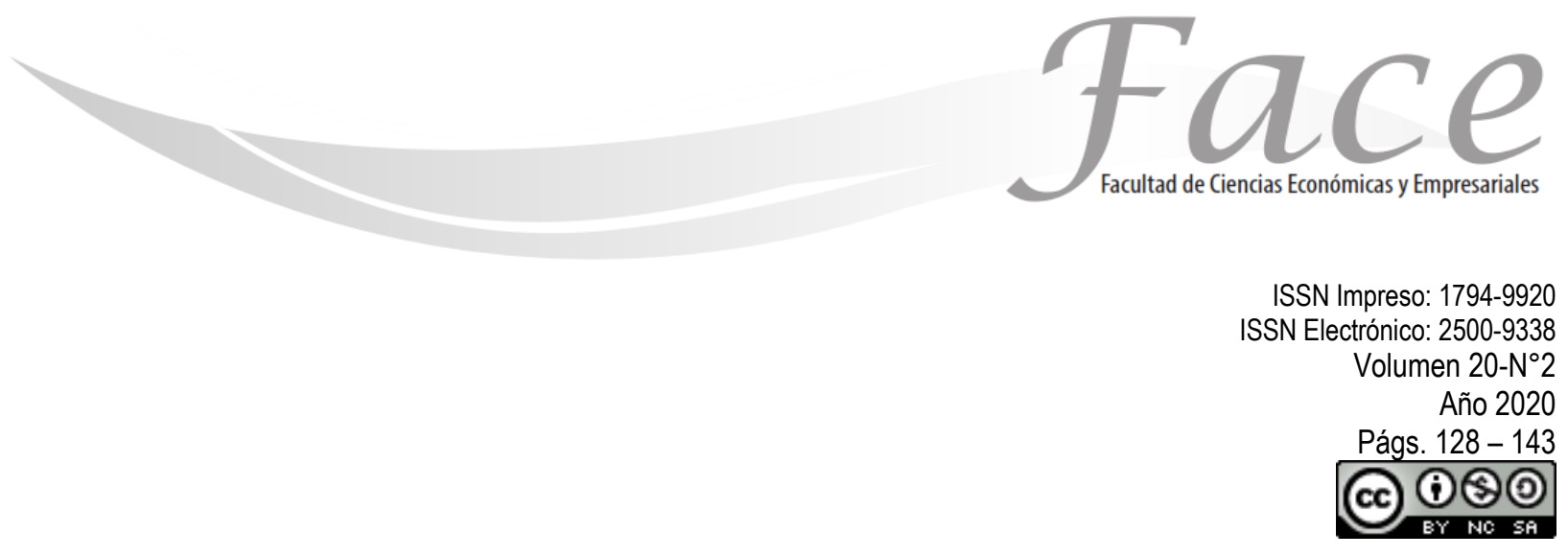

\title{
IMPACTO DE LA CRISIS DE 2008 EN EL CAPITAL INTELECTUAL DE EMPRESAS QUE COTIZAN EN LA BOLSA MEXICANA DE VALORES
}

\author{
Eleazar Villegas González* \\ ORCID: https://orcid.org/0000-0001-8668-1991 \\ Blanca Cecilia Salazar Hernández ${ }^{* *}$ \\ ORCID: http://orcid.org/0000-0001-9427-0826 \\ Jessica Mendoza Moheno** \\ ORCID: https://orcid.org/0000-0003-3947-0256
}

Fecha de Recepción: Octubre 2 de 2020

Fecha de Aprobación: Diciembre 5 de 2020

\section{Resumen:}

Las crisis económicas son procesos que se dan cada cierto tiempo, en algunas ocasiones se consideran como oportunidades de mejora e impulso a formas innovadoras de gestión o por lo contrario impactan negativamente en el desempeño y permanencia de las empresas. Este artículo tiene el objetivo de identificar las diferencias en términos del capital intelectual y competitividad que se generan a partir de la crisis económica 2008 en empresas que cotizan en la bolsa mexicana de valores. A partir de la propuesta de medición capital intelectual VAIC ${ }^{T M}$, se puede observar que los indicadores de capital humano, estructural y empleado se incrementaron después de la crisis, impactando positivamente en la competitividad de las empresas.

Palabras clave: capital humano, competitividad, capital intelectual, capital estructural y capital empleado

'Doctor Eleazar Villegas González. Profesor Investigador del Instituto de Ciencias Económico Administrativas. Universidad Autónoma del Estado de Hidalgo, Mexico . Contacto: evillegas@uaeh.edu.mx.

**Doctora Blanca Cecilia Salazar Hernández. Profesora Investigadora del Instituto de Ciencias Económico Administrativas. Universidad Autónoma del Estado de Hidalgo, Mexico. Contacto: bsalazar@uaeh.edu.mx

***Doctor Jessica Mendoza Moheno. Profesora Investigadora del Instituto de Ciencias Económico Administrativas. Universidad Autónoma del Estado de Hidalgo, Mexico.Contacto: jessica@uaeh.edu.mx 


\title{
IMPACT OF THE 2008 CRISIS ON THE INTELLECTUAL CAPITAL OF COMPANIES LISTED ON THE MEXICAN STOCK EXCHANGE
}

\begin{abstract}
:
Economic crises are processes that occur from time to time, on some occasions they are considered as opportunities for improvement and promotion of innovative forms of management in the other hand, they have a negative impact on the performance and permanence of companies. This article aims to identify the differences in terms of intellectual capital and competitiveness generated from the 2008 economic crisis in companies listed on the Mexican stock market. From the VAICTM intellectual capital measurement proposal, it can be observed that the human, structural and employee capital indicators increased after the crisis, positively impacting the competitiveness of companies.
\end{abstract}

Keywords: human capital, competitiveness, intellectual capital, structural capital and capital employed.

\section{IMPACTO DA CRISE DE 2008 SOBRE O CAPITAL INTELECTUAL DAS EMPRESAS COTADAS NA BOLSA MEXICANA}

\section{Resumo:}

As crises económicas são processos que ocorrem pontualmente, por vezes são considerados oportunidades de melhoria e promoção de formas inovadoras de gestão ou, pelo contrário, têm um impacto negativo no desempenho e permanência das empresas. Este artigo tem como objetivo identificar as diferenças em termos de capital intelectual e competitividade geradas a partir da crise econômica de 2008 nas empresas listadas na bolsa mexicana. A partir da proposta de mensuração do capital intelectual VAICTM, pode-se observar que os indicadores de capital humano, estrutural e de funcionários aumentaram após a crise, impactando positivamente a competitividade das empresas.

Palavras-chave: capital humano, competitividade, capital intelectual, capital estrutural e capital empregado. 


\section{INTRODUCCIÓN:}

Bravo (2012) describe a la globalización como un proceso económico, social y cultural heredado del siglo XX cuyo alcance en todo el planeta hace que todos los países se encuentren interconectados. La interconexión financiera, económica, política, cultural que relaciona a los países, instituciones, pueblos de todo el mundo genera nuevas formas organizativas, nuevas visiones sociales, pero también nuevos problemas en cada uno de estos ámbitos. Uno de esos problemas es sin dudas las crisis financieras.

México no ha sido inmune a estas crisis, a lo largo de su historia las ha experimentado tanto por la globalización como por la mala administración de sus gobiernos.

\section{MARCO TEORICO}

Las crisis financieras

Stiglitz (2012) ha declarado que la globalización tal y como está, no promueve ni la eficiencia ni la equidad global; tanto la globalización del comercio (la circulación de bienes y servicios) como la globalización de los mercados de capitales (la integración internacional de los mercados financieros) han contribuido el aumento de la desigualdad en diferentes maneras, privilegiándose a la renta de capital por encima del capital humano, causando grandes estragos en el ámbito laboral (más de 30 millones de personas sin empleo), además de propiciar una especulación financiera sobre la producción real, generándose así una serie de crisis, que ha llevado a la manifestación de grupos como el 15-M, mejor conocido como el movimiento de los indignados en España, cuyo estandarte fue la protesta en contra del sistema político y económico que los había conducido a una crisis de desempleo, afectando a más del $40 \%$ de la población juvenil (TME, 2009; Rodríguez-Polo, 2013).

Castellanos (2004) ha señalado que las crisis son una característica particular del sistema capitalista, cuyos desajustes han tenido alcances en la economía global, propiciando inestabilidad en el crecimiento y desarrollo de las naciones. Por su parte, Huerta (2010), considera que la crisis representa el fracaso de haber favorecido el libre mercado y reducido la participación del Estado en la regulación y conducción de la actividad económica. Mientras que para Ocampo (2009) parte de la crisis es la descapitalización de las entidades financieras, así como la contracción del crédito y cuyo elemento predominante es el colapso de la actividad productiva en países industrializados.

Entre las crisis más recurrentes (Avendaño, 2000; Requeijo, 2006; Méndez, 2009) se pueden citar las relacionadas con:

- La alimentación mundial: en 2008 el Banco Mundial informó cómo los precios de los alimentos se elevaron a tal punto que la población tuvo que empezar a consumir alimentos más baratos y menos nutritivos.

- Los energéticos: en 1973 con motivo del embargo de la exportación de petróleo de la OPEP a países árabes. En 1990 derivado de la guerra del Golfo pérsico se disparó su precio. En la actualidad su producción ha disminuido por el agotamiento de los combustibles y fósiles, así como su alta demanda.

- Las hipotecas: en el caso de Estados Unidos de América, la conocida como subprime (Ferreira y Marzábal, 2012), estalló a principios de 2007 y se propagó en la economía mundial, la cual 
originalmente afectó el mercado inmobiliario estadounidense y acabó por transformarse en una crisis sistémica global.

- Las financieras: caracterizadas por su gran capacidad de expansión a lo largo de las fronteras geográficas (Kaminsky y Reinhart, 2000), como la del colapso de la burbuja inmobiliaria en Estados Unidos de América, que contaminó el sistema financiero estadounidense con alcances a nivel internacional, teniendo como consecuencia una marcada crisis de liquidez, así como bursátil en los meses de enero y octubre de 2008, conocida como la crisis los países desarrollados.

De estas últimas, han provocado graves daños a la población global (véase figura 1), ya que se han dedicado a contaminar la economía mundial con hipotecas tóxicas. El principio de que "quien contamina, paga" dice que los contaminadores deberían pagar los costes que imponen a los demás, lo cual no se ha aplicado al sistema financiero internacional.

Dicho sistema ha impuesto unas enormes externalidades al resto de la sociedad, los costes totales de la reciente crisis financiera mundial, de la que este sector es sustancialmente responsable, ascienden a varios miles de billones de dólares (Stiglitz, 2012), por lo que ha dejado y continúa dejando marcadas secuelas de desigualdad en los diferentes status de la sociedad mundial, entre ellos al sector empresarial.

Figura 1.

Crisis financieras a nivel mundial

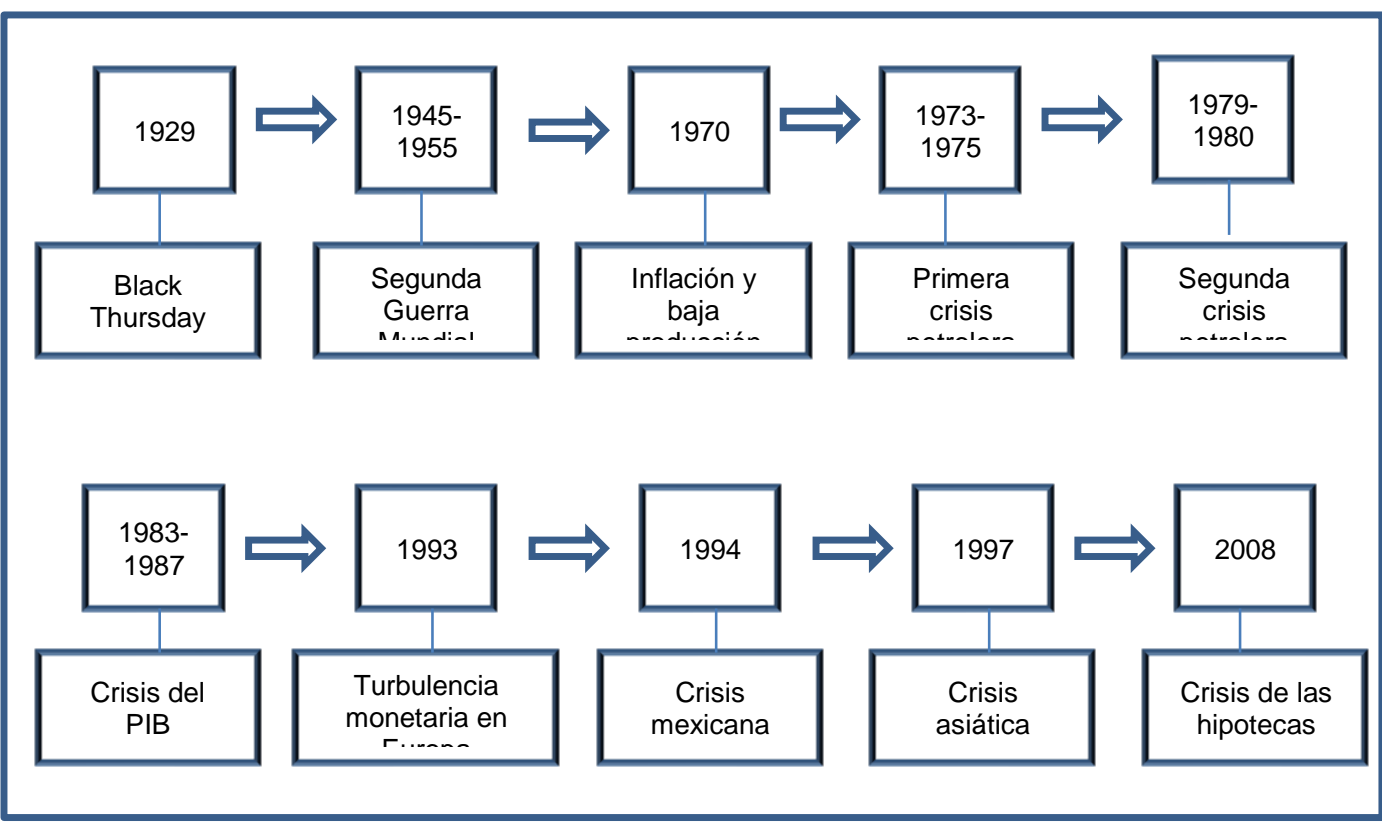

Fuente: Compilación personal basada en Avendaño, 2000; Requeijo, 2006; Méndez, 2009. 
La hipótesis de la inestabilidad financiera de Minsky (1992), apunta que las economías capitalistas experimentan procesos de inflación o deflación por endeudamiento, lo cual se ve reflejado cuando un gran número de empresas llevan a cabo sus operaciones derivadas de una serie de obligaciones de pago heredadas del pasado, y al momento de querer financiar sus operaciones presentes deben de recurrir a obligaciones para el futuro. Esto como resultado de las crisis que a lo largo de la historia les ha golpeado.

\section{Crisis financieras en México}

En el caso de México, tanto los efectos negativos de la globalización como la mala administración de su gobierno han provocado una serie de crisis, como se muestran en la figura 2.

Figura 2.

Crisis financieras en México

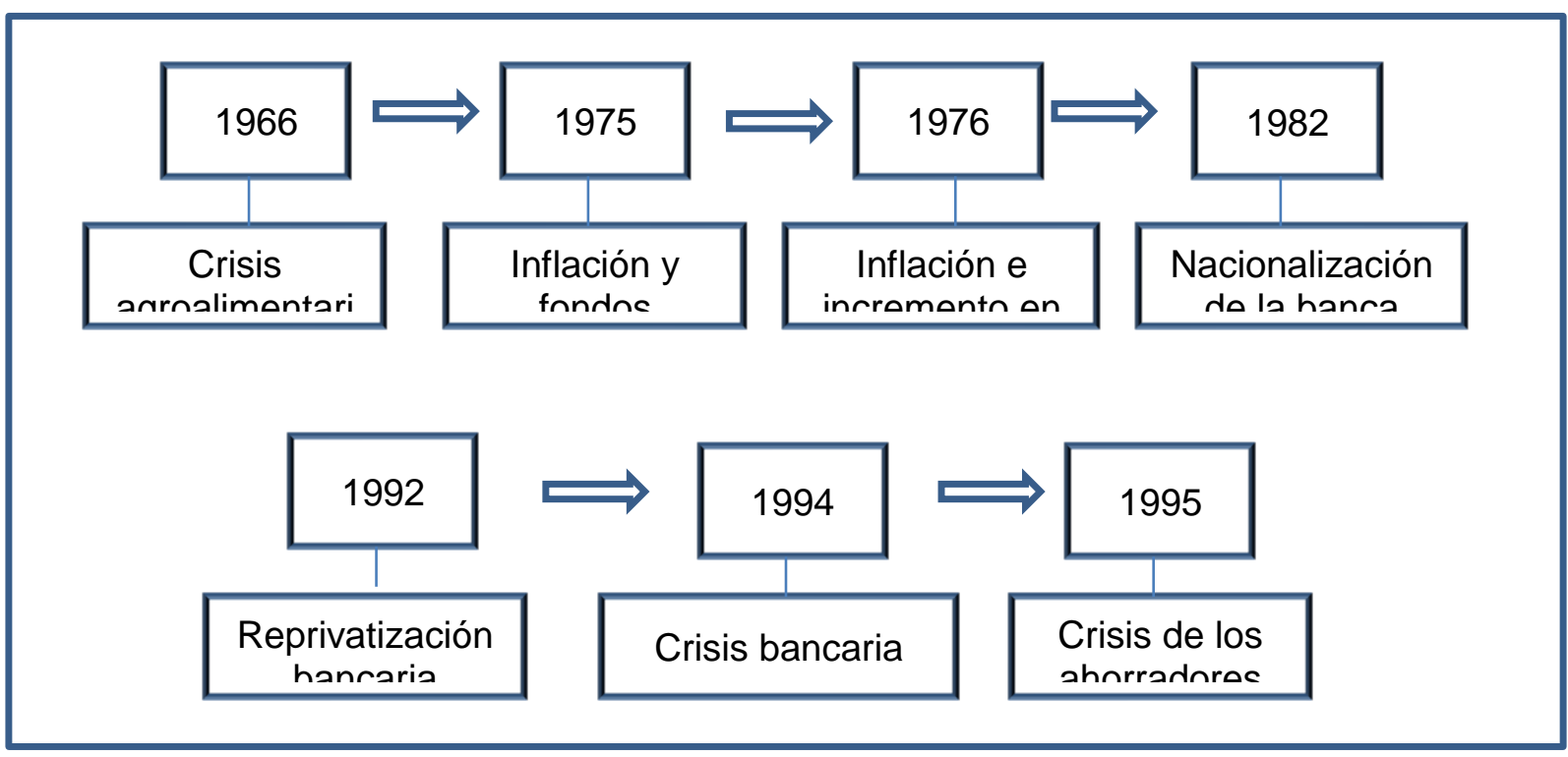

Fuente: Compilación personal basada en González y Aguilar (2003), Villegas y Ortega (2009).

De acuerdo con cifras reportadas por el Banco de México (2012), la crisis financiera mundial de 2008 afectó a México en diversos rubros como fueron la disminución de remesas familiares; la balanza comercial aumento su déficit; se tuvieron bajos niveles en el ingreso promedio real y la masa salarial real; teniendo una fuerte contracción el sector industrial (véase figura 3). 
Figura 3.

Indicadores y determinantes del consumo
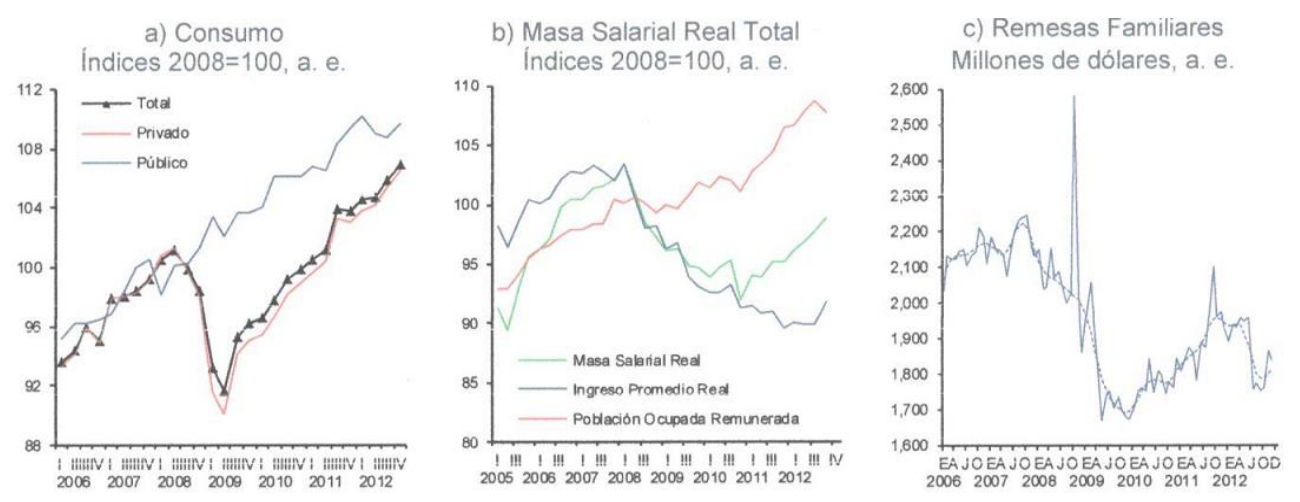

Fuente: Basado en Banco de México (2012), p. 13.

A pesar de la contracción que tuvo el sector industrial mexicano (véase figura 4), éste continúa representando un eje rector de crecimiento y desarrollo ya que emplea materias primas, energía, capital y mano de obra, generando empleos, fortaleciendo las cadenas productivas, incrementando la productividad e impulsando el sector servicios, configurando de esta manera nuevos escenarios de desarrollo económico.
De acuerdo a cifras reportadas por el Banco Mundial (2014) el sector industrial mexicano aportó el 36\% del Producto Interno Bruto PIB en el año 2012. Es por ello que en el presente trabajo se analiza a este sector al momento de medir el capital intelectual en dos periodos, durante la crisis y posterior a la crisis. 


\section{Figura 4.}

Indicadores de la actividad productiva
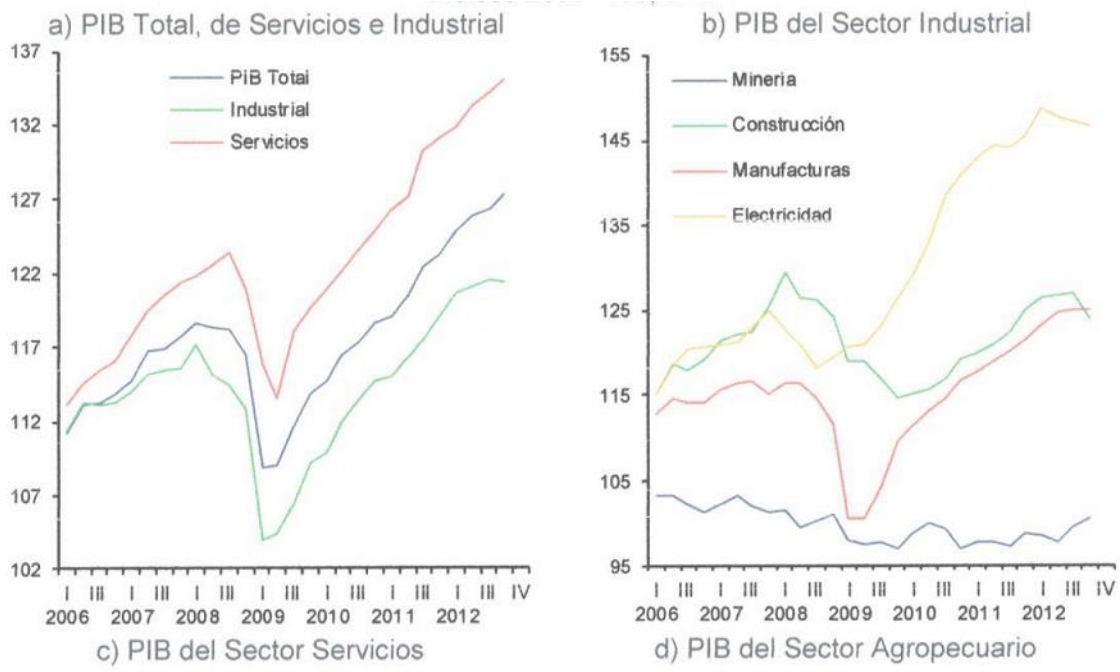

Fuente: Tomado de Banco de México (2012), p.16.

\section{El capital humano}

El capital humano es considerado como el stock de conocimiento individual de una empresa representado por sus empleados (Ordoñez, 2004), los cuales contribuyen a la generación de capital intelectual a través de sus competencias (habilidades y nivel educativo), actitudes (comportamiento de los trabajadores hacia su trabajo) y agilidad mental (característica que les permite a los empleados modificar las prácticas organizativas y desarrollar soluciones innovadoras ante los problemas).
La medición del capital humano

En la medición del capital humano (activos intangibles), Karl-Erik Sveiby (2010) ha identificado cuatro enfoques: el DIC (métodos directos de capital intelectual), MCM (métodos de capitalización de mercados), SC (métodos de cuadros de mando), y, el ROA (método de retorno sobre activos). De este último Sveiby ha agrupado tres modelos, el Economic Value Added EVA ${ }^{\mathrm{TM}}$ desarrollado por Stern and Stewart (1997); el Knowledge Capital Earnings desarrollado por Lev (1999); y el Value Added Intellectual Coefficient VAIC ${ }^{\mathrm{TM}}$ desarrollado por Pulic (1997). Cabe señalar que para la realización de este trabajo de investigación se ha seleccionado el modelo de Pulic para medir el capital intelectual de las empresas cotizadas mexicanas del sector industrial. 


\section{El modelo VAIC ${ }^{\mathrm{TM}}$}

El modelo desarrollado por el profesor y hombre de negocios Ante Pulic $(1998,2000)$ ha servido para medir la eficiencia en la adición de valor a través del capital físico y financiero, el capital humano y el capital estructural con el coeficiente de valor añadido intelectual VAIC ${ }^{\mathrm{TM}}$ en una empresa utilizando las cifras reportadas en la contabilidad, asociadas a cada componente del capital intelectual y el capital empleado (capital físico y financiero). Señalando Pulic que la mayor eficiencia en la creación de valor se basa en el capital humano, como factor de creación de valor decisivo de los negocios modernos. Su idea principal es utilizar el indicador de Valor Añadido VA el cual contribuye a aumentar la productividad y por ende la competitividad en una organización, midiéndolo a través del capital intelectual de los trabajadores.

Dentro de las características que distinguen al modelo $V{ }^{\prime} C^{T M}$ se pueden señalar las siguientes:
- Proporciona una medida estandarizada y una base constante

- Se utiliza información asociada a un selecto grupo de empresas/país

- Involucra indicadores financieros y no financieros que pueden ser fácilmente combinados.

- Todos los datos utilizados en el cálculo del VAIC ${ }^{\mathrm{TM}}$ se basan en información auditada, por lo que los cálculos pueden considerarse objetivos y verificables.

- Es una técnica sencilla que mejora la comprensión cognitiva y permite la facilidad del cálculo.

- La metodología del VAIC ${ }^{\text {TM }}$ se utiliza en más y más estudios captando cada vez más la atención de los investigadores

Pulic (2004), Kujansivu \& Lonnqvist (2007), Laing, Dunn \& Hughes-Lucas (2010) han establecido metodológicamente seis pasos para determiner el valor del VAIC ${ }^{\mathrm{TM}}$, los cuales son explicados en la tabla 1. 


\section{Tabla 1.}

\section{Cálculo de VAICTM}

\begin{tabular}{|c|c|c|c|}
\hline \multirow[t]{3}{*}{ Paso 1: } & \multirow{2}{*}{$\begin{array}{l}\text { cálculo del valor } \\
\text { añadido VA }\end{array}$} & Formula: & Variables: \\
\hline & & $\mathrm{VA}=\mathrm{IT}-\mathrm{CV}$ & $\begin{array}{l}\text { IT }=\text { Ingresos totales } \\
\mathrm{CV}=\text { Costos de ventas }\end{array}$ \\
\hline & Fuente de consulta: & \multicolumn{2}{|c|}{$\begin{array}{l}\text { Estado de pérdidas y ganancias y las notas a los estados } \\
\text { financieros. }\end{array}$} \\
\hline \multirow[t]{3}{*}{ Paso 2: } & \multirow{2}{*}{$\begin{array}{l}\text { cálculo del coeficiente } \\
\text { de eficiencia del } \\
\text { capital humano HCE }\end{array}$} & Formula: & Variables: \\
\hline & & $\mathrm{HCE}=\mathrm{VA} / \mathrm{HC}$ & $\begin{array}{l}\mathrm{VA}=\text { Valor añadido } \\
\mathrm{HC}=\text { Sueldos y salarios (capital } \\
\text { humano) }\end{array}$ \\
\hline & Fuente de consulta: & \multicolumn{2}{|c|}{$\begin{array}{l}\text { Estado de pérdidas y ganancias y las notas a los estados } \\
\text { financieros. }\end{array}$} \\
\hline \multirow[t]{3}{*}{ Paso 3: } & \multirow{2}{*}{$\begin{array}{l}\text { cálculo del coeficiente } \\
\text { de eficiencia del } \\
\text { capital estructural SCE }\end{array}$} & Formula: & Variables: \\
\hline & & $\mathrm{SCE}=\mathrm{SC} / \mathrm{VA}$ & $\begin{array}{l}\mathrm{SC}=\text { Capital estructural } \\
\mathrm{SC}=\mathrm{VA}-\mathrm{HC} \\
\mathrm{VA}=\text { Valor añadido }\end{array}$ \\
\hline & Fuente de consulta: & \multicolumn{2}{|c|}{$\begin{array}{l}\text { Estado de pérdidas y ganancias y las notas a los estados } \\
\text { financieros. }\end{array}$} \\
\hline \multirow[t]{3}{*}{ Paso 4: } & \multirow{2}{*}{$\begin{array}{l}\text { cálculo del coeficiente } \\
\text { de eficiencia del } \\
\text { capital intelectual ICE }\end{array}$} & Formula: & Variables: \\
\hline & & $\mathrm{ICE}=\mathrm{SCE}+\mathrm{HCE}$ & $\begin{array}{l}\text { SCE = coeficiente de eficiencia del } \\
\text { capital estructural } \\
\text { HCE = coeficiente de eficiencia del } \\
\text { capital humano }\end{array}$ \\
\hline & Fuente de consulta: & \multicolumn{2}{|c|}{$\begin{array}{l}\text { Estado de pérdidas y ganancias y las notas a los estados } \\
\text { financieros. }\end{array}$} \\
\hline \multirow[t]{3}{*}{ Paso 5: } & \multirow{2}{*}{$\begin{array}{l}\text { cálculo del coeficiente } \\
\text { de eficiencia del } \\
\text { capital empleado CEE }\end{array}$} & Formula: & Variables: \\
\hline & & $\mathrm{CEE}=\mathrm{VA} / \mathrm{CE}$ & $\begin{array}{l}\mathrm{VA}=\text { valor añadido } \\
\mathrm{CE}=\text { Valor en libros de los activos } \\
\text { netos de una empresa }\end{array}$ \\
\hline & Fuente de consulta: & \multicolumn{2}{|c|}{$\begin{array}{l}\text { Balance General, Estado de pérdidas y ganancias y las notas a los } \\
\text { estados financieros. }\end{array}$} \\
\hline \multirow[t]{3}{*}{ Paso 6: } & \multirow{2}{*}{$\begin{array}{l}\text { cálculo del coeficiente } \\
\text { de valor añadido } \\
\text { intelectual VAIC }^{\mathrm{TM}}\end{array}$} & Formula: & Variables: \\
\hline & & $\mathrm{VAIC}^{\mathrm{TM}}=\mathrm{ICE}+\mathrm{CEE}$ & $\begin{array}{l}\text { ICE = Coeficiente de eficiencia del } \\
\text { capital intelectual } \\
C E E=\text { Coeficiente de eficiencia del } \\
\text { capital empleado }\end{array}$ \\
\hline & Fuente de consulta: & \multicolumn{2}{|c|}{$\begin{array}{l}\text { Balance General, Estado de pérdidas y ganancias y las notas a los } \\
\text { estados financieros. }\end{array}$} \\
\hline
\end{tabular}

Fuente: Elaboración propia siguiendo a Pulic (2004), Kujansivu y Lonnqvist (2007), Laing, Dunn y Hughes-Lucas (2010).

\section{METODOLOGÍA}

El estudio realizado en este artículo es transversal y de tipo cuantitativo, se utilizan datos tomados de informes financieros publicados por las 32 empresas mexicanas que cotizan en la Bolsa Mexicana de Valores (Bolsa Mexicana de Valores, 2014).
A partir de los datos financieros de las empresas, en primera instancia se determinan los coeficientes de capital empleado (CEE), capital humano (HCE) y capital estructural (SCE), así como el coeficiente de de valor añadido intelectual, VAIC ${ }^{\mathrm{TM}}$. Así también, se determinaron las variables proxy de competitividad 0 desempeño (Rendimiento sobre activo total ROA, Market to Book MTB y el Valor agregado por empleado TLP), las cuales son 
utilizadas en para determinar el impacto del capital intelectual en el rendimiento de las empresas estudiadas. Como variables de control se utiliza el tamaño de la empresas mexicanas (SIZE) y el nivel de endeudamiento (DEBT).

Para realizar el estudio, se llevaron a cabo compartivo de medias, estudio de correlación de Pearson y modelos lineales, lo cual permite describir la relación entre las variables de capital intelectual y asi como sus efectos en las varibles proxy de competitividad (Mehri, Umar, Saeidi, Hekmat y NasImosavi, 2013; Mondal y Ghosh, 2012).

Para la validación de los modelos se examinaron los supuestos a través del estadístico Durbin- Watson y el diagnóstico de colinealidad aplicando los estadísticos de tolerancia y los factores de inflación de la varianza (FIV). La base de datos se construyó con datos oficiales registrados en la Bolsa Mexicana de Valores lo cual brinda confiabilidad, validez y objetividad al ser cifras auditadas por firmas reconocidas a nivel nacional e internacional. El análisis estadístico se realiza en el programa SPSS versión 20.

\section{RESULTADOS}

Una vez determinados los coeficientes de capital humano, empleado y estructural, así como el de capital intelectual a través de la metodología del VAIC ${ }^{\text {tm }}$, tanto para 2009 y 2011.
Al realizar la tabla comparativa de medias, se destaca que los indicadores de capital humano, estructural e intelectual muestran una ligera mejoría, no así en el capital empleado (tabla 2).

Así también, las variables proxy de competitividad utilizadas como la ROA, MTB y el TLP presentan una ligera mejoría excepto el MTB lo cual es consistente con la disminución en el capital empleado esto derivado de la disminución del valor de las empresas en el mercado.

\section{Tabla 2.}

Comparativo de medias obtenidas en 2009 y 2011

\begin{tabular}{|l|l|l|}
\hline Rubros & 2009 & 2011 \\
\hline CEE & 0.275 & 0.268 \\
\hline HCE & 3.183 & 3.222 \\
\hline SCE & 0.444 & 0.489 \\
\hline VAIC & 3.902 & 3.979 \\
\hline MTB & 1.743 & 1.608 \\
\hline ROA & 0.033 & 0.058 \\
\hline TLP & 6.247 & 6.448 \\
\hline
\end{tabular}

Fuente: elaboración propia a partir del análisis de datos

Al realizar el estudio de correlación de Pearson, entre las variables propias del VAICTM y las variables proxy de competitividad, se observa que en 2009 existen correlaciones altas y significativas entre el capital humano y el capital intelectual lo cual implica la importancia que tiene el recurso humano en el desarrollo. 
Tabla 3. 2009: Análisis de correlación entre el capital intelectual y variables de competitividad

\begin{tabular}{|l|l|l|l|l|l|l|l|}
\hline Rubro & CEE2009 & HCE2009 & SCE2009 & VAIC2009 & MTB2009 & ROA2009 & TLP2009 \\
\hline CEE2009 & & 0.146 & $.394^{*}$ & 0.26 & $.495^{* *}$ & $.639^{* *}$ & 0.018 \\
\hline HCE2009 & 0.146 & & $.605^{* *}$ & $.988^{* *}$ & $.395^{*}$ & $.609^{* *}$ & $.394^{*}$ \\
\hline SCE2009 & $.394^{*}$ & $.605^{* *}$ & & $.708^{* *}$ & $.476^{* *}$ & $.565^{* *}$ & 0.266 \\
\hline VAIC2009 & 0.26 & $.988^{* *}$ & $.708^{* *}$ & & $.456^{* *}$ & $.671^{* *}$ & $.390^{*}$ \\
\hline MTB2009 & $.495^{* *}$ & $.395^{*}$ & $.476^{* *}$ & $.456^{* *}$ & & $.449^{*}$ & 0.235 \\
\hline ROA2009 & $.639^{* *}$ & $.609^{* *}$ & $.565^{* *}$ & $.671^{* *}$ & $.449^{*}$ & & $.456^{* *}$ \\
\hline TLP2009 & 0.018 & $.394^{*}$ & 0.266 & $.390^{*}$ & 0.235 & $.456^{* *}$ & \\
\hline *. La correlación es significativa en el nivel 0.05 (bilateral). & & & \\
\hline **. La correlación es significativa en el nivel 0.01 (bilateral). & & & \\
\hline
\end{tabular}

Fuente: eleboración propia a partir de los análisis

En este mismo sentido, como lo muestra la tabla 3 existen correlación media alta y significativa entre el capital estructural y el capital humano, así como con el capital intelectual (tabla 3).

Al analizar la relación que guardan los indicadores del capital intelectual con las variables proxy de la competitividad (ROA; MTB y TLP), se destaca, la relación que guarda el VAICTM como coeficiente de capital intelectual con el rendimiento, el valor de capitalización del mercado y la productividad por trabajador.

Así también, existe correlación significativa entre los indicadores (HCE, CEE y el SCE) que integran el VAIC ${ }^{\mathrm{TM}}$ con la ROA y el MTB, así no con la productividad que únicamente se relaciona significativamente con el capital humano.

Al realizar el análisis de correlación de los indicadores de 2011, se observa la misma tendencia, con excepción de la falta de correlación entre el capital estructural y el rendimiento financiero $(R O A)$ y la relación existente entre el capital estructural y la productividad (TLP).

En resumen, el análisis de correlación nos permite concluir la importancia que tiene los intangibles en el rendimiento financiero de las empresas. Aunque la productividad por empledo en algunos casos no guarda relación. 
Tabla 4. 20011: Análisis de correlación entre el capital intelectual y variables de competitividad

\begin{tabular}{|l|l|l|l|l|l|l|l|}
\hline Rubro & CEE2011 & HCE2011 & SCE2011 & VAIC2011 & MTB2011 & ROA2011 & TLP2011 \\
\hline CEE2011 & & 0.205 & $.433^{*}$ & 0.302 & $.614^{* *}$ & $.537^{* *}$ & 0.072 \\
\hline HCE2011 & 0.205 & & $.760^{* *}$ & $.992^{* *}$ & $.420^{*}$ & $.428^{*}$ & $.419^{*}$ \\
\hline SCE2011 & $.433^{*}$ & $.760^{* *}$ & & $.823^{* *}$ & $.481^{* *}$ & 0.321 & $.432^{*}$ \\
\hline VAIC2011 & 0.302 & $.992^{* *}$ & $.823^{* *}$ & & $.473^{* *}$ & $.455^{* *}$ & $.429^{*}$ \\
\hline MTB2011 & $.614^{* *}$ & $.420^{*}$ & $.481^{* *}$ & $.473^{* *}$ & & $.445^{*}$ & 0.334 \\
\hline ROA2011 & $.537^{* *}$ & $.428^{*}$ & 0.321 & $.455^{* *}$ & $.445^{*}$ & & $.394^{*}$ \\
\hline TLP2011 & 0.072 & $.419^{*}$ & $.432^{*}$ & $.429^{*}$ & 0.334 & $.394^{*}$ & \\
\hline *. La correlación es significativa en el nivel 0.05 (bilateral). & & & \\
\hline **. La correlación es significativa en el nivel 0.01 (bilateral). & & & \\
\hline
\end{tabular}

Fuente: eleboración propia a partir de los análisis

Se han diseñado 2 modelos lineales uno para 2009 y otro para 2011 con el fin de establecer relación que existe entre el índice del VAIC ${ }^{T M}$ y el indicador de rentabilidad (ROA). teniendo como variables de control el tamaño y la deuda.
Como se observa en la tabla 5. en el modelo 2009 las variables involucradas. es decir el índice de capital intelectual. empleado y estructural así como la deuda y el tamaño explica en su conjunto el $74.9 \%$ de la varianza. mientras que en el caso del modelo 2011 lo explican 45.3\% (tabla 5).

Tabla 5. Modelos de regresión lineal de los años 2009 y 2011

\begin{tabular}{|c|c|c|c|c|c|c|}
\hline \multicolumn{7}{|c|}{ Resumen del modelo } \\
\hline \multirow[t]{2}{*}{ Modelo } & \multirow[t]{2}{*}{$\mathrm{R}$} & \multirow[t]{2}{*}{ R cuadrado } & \multirow{2}{*}{$\begin{array}{c}\mathrm{R} \\
\text { cuadrado } \\
\text { ajustado }\end{array}$} & \multirow{2}{*}{$\begin{array}{c}\text { Error estándar de la } \\
\text { estimación }\end{array}$} & \multicolumn{2}{|c|}{ Estadísticos de cambio } \\
\hline & & & & & $\begin{array}{c}\text { Cambio en } \mathrm{R} \\
\text { cuadrado }\end{array}$ & Cambio en $\mathrm{F}$ \\
\hline 2009 & $.893^{a}$ & 0.798 & 0.749 & 0.038 & 0.798 & 16.434 \\
\hline 2011 & $.747^{a}$ & 0.559 & 0.453 & 0.057 & 0.559 & 5.276 \\
\hline
\end{tabular}

Fuente: elaboración propia

a. Predictores: (Constante). SIZE, DEUDAS, SCE, CEE, TLP y HCE. Variable dependiente: ROA

En la tabla 6 se presentan los resultados obtenidos de los coeficientes de regresión lineal de los 2 modelos, los cuales explican el impacto que tienen los índices de capital intelectual en en la competitividad de la empresa (ROA). 
En el caso del modelo 2009, la regresión lineal explica que la relación entre la variable de rendimiento (ROA) como dependiente, se relaciona positiva y significativamente con el capital empleado $\left(\beta=.152^{*}\right)$, así como, con el capital humano $\left(\beta=.008^{\star *}\right)$, mientras que con la deuda se relaciona negativamente $\left(\beta=-0.94^{* *}\right)$. Las variables capital empleado y tamaño no son significativas por lo que en este caso no iniceden en la competitividad de la empresa expresada a través de la ROA.

En los resultados del Modelo 2011, se observa que variables que contribuyen al rendimiento (ROA) son el capital empleado $\left(\beta=.212^{*}\right)$, el capital humano $\left(\beta=.014^{\star *}\right)$ y el capital estructural $\left(\beta=-.096^{* * *}\right)$, tanto el tamaño como la deuda no se consideran como variables de impacto.

Tabla 6. Modelos de regresión lineal de los años 2009 y 2011

\begin{tabular}{|c|c|c|c|c|c|c|}
\hline \multirow{2}{*}{\multicolumn{2}{|c|}{ Modelo }} & \multirow{2}{*}{\multicolumn{2}{|c|}{ Coeficientes no estandarizados }} & Coeficientes & \multirow[t]{2}{*}{$\mathrm{t}$} & \multirow[t]{3}{*}{ Sig. } \\
\hline & & & & estandarizados & & \\
\hline \multirow{7}{*}{2009} & (Conctanta) & & & & & \\
\hline & & & & & & \\
\hline & & & .032 & .401 & 4.001 & .000 \\
\hline & HCE2009 & .008 & .003 & .317 & 2.418 & .023 \\
\hline & SCE2009 & 017 & .022 & .099 & .765 & .451 \\
\hline & DEUDAS2009 & -.094 & .039 & -.244 & -2.433 & .022 \\
\hline & SIZE2009 & 166 & .253 & .073 & 659 & .516 \\
\hline \multirow[t]{6}{*}{2011} & (Constante) & -.105 & .095 & & -1.106 & .279 \\
\hline & CEE2011 & .212 & .062 & .557 & 3.434 & .002 \\
\hline & HCE2011 & .014 & .007 & .477 & 2.121 & .044 \\
\hline & SCE2011 & -.096 & .056 & -.423 & -1.711 & .099 \\
\hline & DEUDAS2011 & -.064 & .060 & -.159 & -1.067 & .296 \\
\hline & SIZE2011 & .122 & .259 & .078 & .473 & .640 \\
\hline
\end{tabular}

Fuente: elaboración propia

b. Predictores: (Constante). SIZE, DEUDAS, SCE, CEE, TLP y HCE

c. Variable dependiente: ROA

* La correlación es significativa al nivel:0.01

** La correlación es significativa al nivel:0.05

${ }^{* * *}$ La correlación es significativa al nivel:0.1

Tabla 7 Estadístico de independencia Durbin-Watson

Los modelos lineales presentados cumplen con la validez requerida. El estadístico Durbin-Watson (tabla7) que valida el grado de independencia que existe entre los residuos, se observa que al ser cercanos a 2 no existe autorrelación asegurando la independencia de las variables predictoras, validando dicho supuesto.

\begin{tabular}{|c|c|c|c|}
\hline Modelo & $F$ & Sig. & Durbin-watson \\
\hline 2009 & 16.434 & $.000^{\mathrm{b}}$ & 1.897 \\
\hline 2011 & 5.276 & $.001^{\mathrm{b}}$ & 1.496 \\
\hline
\end{tabular}

Fuente:eleaboración propia a partir de análisis en SPSS 
Por otro lado, el diagnóstico de colinealidad ((tabla 8), realizado con los estadísticos de tolerancia y los FIV reflejan tolerancias mayores a 0.10 y los valores del FIV menores a 10 , por lo que se concluye que no existe relación lineal exacta entre las variables independientes.

Tabla 8 Diagnóstico de colinealidad, tolerancia y FIV

\begin{tabular}{|c|c|c|c|}
\hline \multirow{2}{*}{\multicolumn{2}{|c|}{ Modelo }} & \multicolumn{2}{|c|}{$\begin{array}{c}\text { Estadísticas de } \\
\text { colinealidad }\end{array}$} \\
\hline & & Tolerancia & VIF \\
\hline \multirow{6}{*}{$\begin{array}{l}2009 \\
\text { ROA }^{a}\end{array}$} & (Constante) & .749 & 1.336 \\
\hline & CEE2009 & .470 & 2.129 \\
\hline & HCE2009 & .485 & 2.062 \\
\hline & SCE2009 & .575 & 1.738 \\
\hline & $\begin{array}{l}\text { DEUDAS200 } \\
9\end{array}$ & .656 & 1.524 \\
\hline & SIZE2009 & .749 & 1.336 \\
\hline \multirow{6}{*}{$\begin{array}{l}2011 \\
\text { ROA }^{a}\end{array}$} & (Constante) & & \\
\hline & CEE2011 & .670 & 1.493 \\
\hline & HCE2011 & .348 & 2.870 \\
\hline & SCE2011 & .289 & 3.461 \\
\hline & $\begin{array}{l}\text { DEUDAS201 } \\
1\end{array}$ & .796 & 1.257 \\
\hline & SIZE2011 & .654 & 1.528 \\
\hline
\end{tabular}

Fuente:eleaboración propia a partir de análisis en SPSS

a. Variable dependiente: ROA

\section{CONCLUSIONES}

Se observa que tanto los indicadores del VAIC como de competitividad se incrementaron después de la crisis efectivamente las crisis impactan el capital intelectual en las organizaciones.

Existe una fuerte correlación entre los índices de capital intelectual y las variables proxy de competitividad utilizadas como la ROA y MTB, con excepción de 2011 donde la correlación con el Capital Empleado no es significativa.

En cuanto al resultado de los modelos lineales, en 2009 , los aspectos que impactan en el rendimiento de las empresas son: capital humano, el capital empleado, el valor agregado por empleado y la deuda.

Mientras que en 2011, los aspectos que impactan en el rendimiento de las empresas son: capital humano, el capital empleado, el capital estructural, el valor agregado por empleado y el tamaño. El regresor Deuda no es significativo

\section{REFERENCIAS:}

Avendaño, O. (2000). El sistema financiero internacional, instituciones y crisis financieras. México, D.F. Editorial Porrúa.

Banco de México (2012). Informe anual 2012. Disponible en: http://www.banxico.org.mx/publicaciones-ydiscursos/publicaciones/informesperiodicos/anual/\%7B8E01B9AD-971C-FEF056A4-DA54C7106DF8\%7D.pdf

Banco Mundial (2008). Crisis precios de alimentos. Disponible en: http://www.bancomundial.org/temas/preciosali $\underline{\text { mentos/iniciativas.htm }}$ 
Banco Mundial (2014). Indicadores de desarrollo mundial. México, América Latina y el Caribe [en línea; consultado 24 Jun 2014]. Disponible en: Disponible

en: http://datos.bancomundial.org/pais/mexico

Bravo, E. (2012). Globalización, innovación tecnológica y pobreza. Aproximación a las nuevas conceptualizaciones en Latinoamérica. (Spanish). Espacio Abierto. Cuaderno Venezolano De Sociología, 21(3), 543-556. Disponible en: http://web.ebscohost.com/ehost/pdfviewer/pdfv iewer?sid=9423f28a-78c4-47aa-b3ec$314 \mathrm{fd} 5 \mathrm{f} 7 \mathrm{f025} \%$ 40sessionmgr $115 \&$ vid=4\&hid=1 $\underline{14}$

Castellanos, R. (2004). La crisis económica global en los marcos de la globalización neoliberal. Santiago, no. 105, 2004, p. 114. Disponible en: https://go.gale.com/ps/anonymous?id=GALE\% 7CA169960825\&sid=googleScholar\&v=2.1\&it= r\&linkaccess=abs\&issn=00489115\&p=IFME\&s $\mathrm{w}=\mathrm{w}$

Ferreira Jr., V. M., \& Marzábal, Ó. R. (2012). La crisis financiera global en perspectiva: génesis y factores determinantes. (Spanish). Revista De Economía Mundial, (31), 199-226.

González, P. y Aguilar, H. (2003). México ante la crisis I. México, DF. Siglo XXI editores.

Guerras, L y Navas, J. (2007). La dirección estratégica de la empresa. Teoría y aplicaciones. Thomson-Reuters Civitas, Madrid.
Huerta, A. (2010). Las lecciones no aprendidas d la crisis en México. Metropolita. No. 61. Disponible en: https://snt150.mail.live.com/mail/ViewOfficePre view.aspx?messageid=mgoyeURSPP5BGdNg AjfeScgg2\&folderid=flinbox\&attindex $=2 \& c p=-$ 1\&attdepth $=2 \& n=33281751$

Jensen, M. y Meckling, W. (1976), "The Theory of the Firm: Managerial Behaviour, Agency Costs, and Ownership Structure", Journal of Financial Economics, vol. 3, n 4, pp. 305-360.

Kaminsky, G. \& Reinhart, C. (2000). On crises, contagion, and confusión. J. Int. Econ., 51 (1) (2000), pp. 136-145. Disponible en: $\quad$ https://doi.org/10.1016/S0022$\underline{1996(99) 00040-9}$

Kujansivu, P. \& Lonnqvist, A. (2007), "Investigating the value and efficiency of intellectual capital", Journal of Intellectual Capital, Vol. 8 No. 2, pp. 272-287.

Laing G., Dunn, J \& Hughes-Lucas, S. (2010). Applying the VAIC ${ }^{\mathrm{TM}}$ model to Australian Hotels, Journal of Intellectual Capital, 11(3), 269-283. Disponible en: http://www.emeraldinsight.com.pbidi.unam.mx: 8080/search.htm?PHPSESSID=n0j8m3gu56m v4r29sipdj8sjs2\&st1=Applying+the+VAIC\%E2 $\% 84 \% \mathrm{~A} 2+$ model+to+Australian+Hotels $\% 2 \mathrm{C}+\&$ $\mathrm{ct}=\mathrm{all} \& \mathrm{ec}=1 \& \mathrm{bf}=1 \& \mathrm{go}=\mathrm{Go}$

Méndez, J. (2009). Fundamentos de economía. México, D.F. McGraw-Hill. 
Minsky, H. (1975). John Maynard Keynes. Columbia University Press.

Minsky, H. (1992). The Financial Instability Hypothesis. Working Paper No. 74.

Ocampo, J. (2009). Impactos de la crisis financiera mundial sobre América Latina. Revista de la Cepal Número 97, 4.

Ordóñez (2004). El capital estructural organizativo como fuente de competitividad empresarial. Disponible en: www.minetur.gob.es/Publicaciones/.../357/13 PatriciaOrdonez 357

Pulic, A. (1998). Measuring the performance of intellectual potential in knowledge economy. Paper presented at the 2nd World Congress on Measuring and Managing Intellectual Capital, McMaster University, Hamilton.

Pulic, A. (2000). VAIC ${ }^{\text {TM }}$ - an accounting tool for IC management. International Journal of Technology management, 20(5), 702-714. Disponible en: http://resources.metapress.com/pdfpreview. axd?code=y2c0e45|6wb4a9|f\&size=lar gest

Pulic, A. (2004), Intellectual capital - does it create or destroy value?, Measuring Business

Excellence, Vol. 8 No. 1, pp. 62-8. Disponible en:

http://www.emeraldinsight.com/journals.htm?ar ticleid $=843767$
Ross, A. (1973). The Economic Theory of Agency: The Principal's Problem. American Economic Review, vol. 63, n² 2, pp. 134-139.

Requeijo, J. (2006). Anatomía de las Crisis

Financieras. Madrid. McGraw-Hill.

Rodríguez-Polo, X. R. (2013). Bloqueo mediático, redes sociales y malestar ciudadano. Para entender el movimiento español del 15-M. (Spanish). Palabra Clave, 16(1), 45-68.

Stiglitz, J. (2012). El precio de la desigualdad. México. Santillana Ediciones.

Sveiby, K-E. (2010). Methods for Measuring Intangible Assets. Copyright @Karl-Erik Sveiby Jan 2001, updated 27 April 2010. All rights reserved.

Disponible en:

http://www.sveiby.com/articles/IntangibleMetho ds.htm

Tendencias Mundiales del Empleo TME (2009). Crisis financiera mundial: Espectacular aumento del desempleo, de los trabajadores pobres y de los empleos vulnerables debido a la crisis económica mundial. (Spanish). (2009). Trabajo - La Revista de la OIT, trabajo número 65 44-45

Villegas, E. y Ortega, R. (2009). Sistema financiero de México. México, D.F. Editorial McGrawHill 